\title{
ROSE: Alternative for Cancelled and Inconclusive Cytopathologic Examinations, as Well as Professional Training at the UNESP-Botucatu Veterinary Hospital
}

\author{
Fabiane Andrade Correia Neiva1, Eduardo Eburnio1, Paula de Sanctis ${ }^{2^{*}(\mathbb{D} \text {, }}$ \\ Nayara Maria Gil Mazzante ${ }^{1}$, Noeme Sousa Rocha ${ }^{1}$ \\ ${ }^{1}$ Investigative and Comparative Pathology Laboratory, SÃo Paulo State University (UNESP), Botucatu, Brazil \\ ${ }^{2}$ School of Medicine from Botucatu, SÃo Paulo State University (UNESP), Botucatu, Brazil \\ Email: *paulasanctis@gmail.com
}

How to cite this paper: Neiva, F.A.C., Eburnio, E., de Sanctis, P., Mazzante, N.M.G. and Rocha, N.S. (2019) ROSE: Alternative for Cancelled and Inconclusive Cytopathologic Examinations, as Well as Professional Training at the UNESP-Botucatu Veterinary Hospital. Open Journal of Veterinary Medicine, 9, 121-135.

https://doi.org/10.4236/ojvm.2019.99011

Received: July 24, 2019

Accepted: September 21, 2019

Published: September 24, 2019

Copyright $\odot 2019$ by author(s) and Scientific Research Publishing Inc. This work is licensed under the Creative Commons Attribution International License (CC BY 4.0).

http://creativecommons.org/licenses/by/4.0/

cC) (7) Open Access

\begin{abstract}
ROSE is a method for fast sample evaluation that does not compromise sensitivity and specificity in the hospital environment when establishing the diagnosis and the therapeutic protocol. The technique is already well-established in human medicine as it operates within the standard operational procedure, reducing the rates of inconclusive results and cancelled cytopathology assays in the hospital environment. However, its application is scarce in Veterinary Medicine, including in hospitals where intensive care is available. This study aims at conducting a case-by-case assessment of inconclusive and cancelled examinations in the Cytopathology Outpatient Clinic at UNESP (HV), Botucatu, Brazil, from 2012 to 2016 and ascertains the causes. For this purpose, a retrospective study was conducted for 9587 examinations in canines, of which $4.1 \%$ and $10.44 \%$ were inconclusive and cancelled, respectively. These results are not in line with ROSE, which foresees a total rate of 5\% for these occurrences. The reasons for these high rates in the outpatient clinic were the lack of a Standard Operating Procedure (SOP) and the relative inexperience of the residents in a university hospital. Therefore, with the adoption of the ROSE protocol for veterinary cytopathological examinations, together with adequate training for the outpatient professionals responsible for the examinations, the goals set forth by the ROSE protocol would be met and the rates of inconclusive and cancelled examinations would decrease considerably. Lastly, this study hopes to contribute towards the diagnosis and therapeutic protocols of the main diseases affecting dogs.
\end{abstract}




\section{Keywords}

PAAF, Inconclusive or Canceled Cytology, Canine, ROSE, Rapid On-Site Evaluation

\section{Introduction}

The ongoing quest for quality in scientific activities requires training and mastery of the knowledge required for these activities to be conducted in a way that assures precision, validity and quality in the results, as well as the integrity of personnel and facilities [1]. Several initiatives have based themselves on methodologies and tools that are widely employed in other business, such as Total Quality Management, Lean Thinking, Six Sigma, Process Redesign, ISO Guidelines or Quality Awards [1] [2].

Histopathology and cytopathology are examinations that allow the differentiation of benignant injuries from malignant ones according to the following criteria: morphology; homogeneity; conformation; differentiations such as the degree of anisocytosis, basophilia, mitotic activity, cellular edges, vacuoles, granules, nucleoli, nucleus-to-cytoplasm ratio, as well as alterations such as necrosis and inflammation. Therefore, a cytopathological report should contain a well-designed description [3] [4] [5] [6] [7].

Factors that may interfere and complicate the examination include: heterogeneous composition of tumors, incomplete clinical history, lack of standardization in the methodology, inter-observer variation, irregular distribution, cystic nature, small injuries, difficulties during the collection and presence of necrosis and inflammation [3] [4] [8]. As such, in about $5 \%$ to $15 \%$ of the cases, the Fine Needle Aspiration (FNA) biopsy did not reach a conclusive diagnosis, not because of the technique itself, but rather because of the inexperience of the pathologist or the characteristics of the injury [4] [8] [9].

Therefore, an alternative to increase quality in these cases is applying the ROSE (Rapid On-Site Evaluation) protocol, which consists in establishing standards to assure the sensitivity and specificity of the cytopathological exam without compromising its inherent quickness, considering all stages of the process, from requisition, collection, processing, reading and interpretation to issuing the diagnosis, as well as the determining of the previous classification of the injury [10].

In human medicine, the ROSE protocol has proved to be efficient in several areas that are similar to FNA, particularly for costly, image-guided procedures, such as transbronchial endoscopy and organ ultrasound. In addition, the ROSE protocol potentializes the sensitivity and specificity in outpatient clinics, where there are a high number of biopsies with inconclusive results or cancelled biopsies due to an inadequate use of the technique [11] [12] [13] [14].

For the implementation to be efficient, stages should be established through a protocol with guidelines, with a clinical examination, anamnesis and the macros- 
copic and microscopic of each injury representing important starting points [15] [16]. Then, it is important to choose an adequate technique to collect the material, assessing color, size, quantity, temperature, consistence and odor, all of which are directly linked with a successful definitive diagnosis or the need for complementary examinations [17] [18] [19].

The FNA technique, in order to be applied correctly, should consider factors such as collection with or without aspiration, the number of slides, the availability of a slide rack for protection, asepsis, isolation of the field, choice of syringe and needle diameter, volume of material in the slide and presence of artifacts, particularly when preparing and staining the slides [17] [20]. Therefore, for each collection, three to four slides should be prepared: one dried naturally and the remaining three fixed in a $95 \%$ ethanol solution or a $10 \%$ buffered formalin solution [5].

Considering that the main goal of this procedure is to be more dynamic and agile, the staining methods should find a happy medium between speed, ease of preparation and quality [11] [12]. Professionals should be familiar with the methods more commonly employed with the ROSE protocol and their particularities in order to potentialize their characteristics in each type of injury and favorably impact the final diagnosis. The combination of two staining techniques with different affinities (nucleus and cytoplasm) is also something that should be considered, since doing so provides a better basis for the diagnosis and improves its efficacy, as is the case with the combination of the Giemsa and Papanicolaou stains [21]. The availability, calibration and maintenance of equipment are of utmost importance for the success of the procedure [13] [22].

After ascertaining that the fundamentals have been duly established, it is necessary to assess the expression of the sample, ensuring that the examinations are conducted in an adequate and systematic way in order to reach maximum diagnostic certainty [22] [23] [24]. The quality of the sample increases as a professional observes or conducts more procedures, ensuring that the material is used more efficiently and, therefore, reducing the number of cancellations, inadequate samples and inconclusive results [25]. As the ROSE protocol reduces the number of inadequate samples, it also reduces the need to repeat a procedure and, therefore, the costs and the stress caused to the patient [26] [27] [28].

Kubota et al. (2017) have shown the importance of the correlation between past and current data during the procedures in cases of Inflammatory Mammary Carcinoma, in which the use of FNA was restricted due to the absence of neoplastic cells in the lymphatic vessels at the dermis. However, the diagnosis was possible based on a complete clinical examination and on the assessment of the animal's profile. Nasuti et al. (2002) have employed the ROSE protocol for FNA biopsies, saving approximately US\$404,525 per year, eliminating the need to repeat procedures, which is also in line with the report by Selhi et al. (2018).

The ROSE protocol may also be employed together with digital cytopathology in order to speed up the diagnostic process, and optimize work and procedure 
times, particularly in locations with high demand for cytopathological assays or high manpower rotativity. Therefore, it is important to emphasize that, even when using electronic microscopy and other imaging procedures, the ROSE protocol is extremely important due to the high costs involved [29] [30]. As possible disadvantages, some pathologists have mentioned time restraints, interruption of the work flow and bad use of the available resources when more than a single professional is necessary [31].

Finally, this study aims at cataloguing and discussing the factors that may lead a cytopathological examination to be inconclusive or cancelled in the routine of our veterinary hospital, as well as developing a Veterinary ROSE Protocol to reduce such occurrences based on the advantages and scientific criteria established by the use of ROSE in humans, resulting in a service with a proper quality control and higher credibility for FNAP.

\section{Material \& Methods}

\section{Study Regulations}

The study was approved by the Ethics Committee on Animal Use (CEUA, Comitê de Ética no Uso de Animais) of the School of Veterinary Medicine and Animal Sciences, UNESP, Botucatu, Brazil, under protocol number 0087/2017. This study considered all reports from cytopathological assays, following a retrospective and systematic analysis in a digital database of all cases treated at the Cytopathology Outpatient Clinic of the UNESP Veterinary Hospital, Botucatu, Brazil, between 2012 and 2016 .

In order to establish a standard for data collection, the study assessed the following criteria: Diagnosis; Macroscopic Description and Microscopic Description of each cytopathological assay. Pathologically, the assays were classified as: Neoplasms; Inflammations; Other Injuries; and Cancelled/Inconclusive. Within the Cancelled/Inconclusive group, another subclassification was established separating Inconclusive diagnoses from Cancellations.

All data was organized in tables using Microsoft Excel ${ }^{\circ}$ so that descriptive statistics could be applied and charts created. From this data, the samples were stratified through a quantitative descriptive analysis and estimated as absolute values and percentages for each item within each group, allowing statistical inference to be used to establish an adequate ROSE Protocol for the canine population and, by extrapolation, for other animal species.

\section{Results \& Discussion}

According to the survey conducted (Table 1), the 6162 requisitions originated 10,212 FNA biopsies during the 5 years analyzed. Canines represented approximately $94 \%$ of these examinations (9587), of which $4.1 \%$ were inconclusive and $10.44 \%$ were cancelled, which is in line with the study conducted by Selhi et al. (2018). Aside from 2012, the remaining years analyzed were within the results observed in the literature of about 15\% [3] [5] [7] [32] [33] [34]. 
Table 1. Relationship between the number of cytopathological exam requisitions (FNA), the number of exams conducted and the rate of inconclusive or cancelled exams (\%) at the HV-FMVZ between 2012 and 2016.

\begin{tabular}{cccc}
\hline Year & FNA Requisitions & Exams conducted & Inconclusive and cancelled (\%) \\
\hline 2012 & 1140 & 2024 & 23.07 \\
2013 & 1296 & 2159 & 14.27 \\
2014 & 1177 & 1831 & 10.54 \\
2015 & 1199 & 1827 & 14.10 \\
2016 & 1350 & 2371 & 13.41 \\
Total & 6162 & 10,212 & 14.96 \\
\hline
\end{tabular}

The injuries diagnosed in the dogs through FNA were inflammation, malignant and benignant neoplasms, hyperplasia, dysplasia, metaplasia, effusions (cysts, ascites, edemas) and "others". Regarding the number of cancelled or inconclusive examinations, there was a reduction from 2012 to 2015, with a slight increase in 2016 (Figure 1).

As shown in Figure 1, the most common injuries diagnosed were neoplasms, and this prevalence highlights the importance of a quick, precise, reliable, minimally-invasive and low-cost diagnosis, as well as stimulates the search for new quality control strategies for the diagnostic process. In addition, the significant number of cancelled and inconclusive exams is an alert regarding the importance of possible errors during the examination or unfamiliarity with the technique, both of which have been described by Murussi et al. (2001), Sun et al. (2015), Cascone et al. (2017) and Dolka et al. (2018).

From this data, it is possible to note a wide array of factors associated with cancelled or inconclusive exams (Table 2 and Table 3), as well as variations in their intercurrences and difficulties in standardizing nomenclatures and classifying the injuries, which may be related to the rotativity of professionals and their varying degrees of instruction considering that the study was intentionally conducted at an university hospital in order to highlight the importance of proper operator qualification and of a Quality Control Protocol in the cytopathological diagnosis.

Cases of "insufficient material", which was the most prevalent reason in this study, recorded the following rates from 2012 to 2016: 9.5\%, 29.35\%, 28.41\%, $44.92 \%$ and $37.97 \%$. This reason was recorded when the sample collected was not sufficient to conduct the examination, and usually happens due to operator inexperience, incorrect choice of collection technique (puncture, imprint, exfoliative or liquid analysis), incorrect delimitation of the injury, mistakes when processing the sample or due to the characteristics and presentation of the injury, which could be insufficiently exfoliative, resulting in insufficient material for the diagnosis [3] [4] [17] [35].

Cases of "undetermined diagnosis" recorded the following rates from 2012 to 2016: $52.05 \%, 43.68 \%, 48 \%, 23.81 \%$ and $21.74 \%$. This category included incomplete descriptions and lack of correct pathological classification, such as "presence 
Table 2. Factors influencing inconclusive results in canine cytopathological exams at the HV-UNESP-Botucatu from 2012 to 2016.

\begin{tabular}{cccccc}
\hline Reasons for Inconclusive Results & \multicolumn{7}{c}{ Year } \\
\cline { 2 - 6 } & $\mathbf{2 0 1 2}$ & $\mathbf{2 0 1 3}$ & $\mathbf{2 0 1 4}$ & $\mathbf{2 0 1 5}$ & $\mathbf{2 0 1 6}$ \\
\hline Contamination by blood & 1 & 1 & 9 & 36 & 34 \\
Undetermined diagnosis & 89 & 38 & 12 & 15 & 10 \\
Inflammatory process & 12 & 9 & 1 & 0 & 0 \\
Negative for neoplasm & 67 & 39 & 3 & 2 & 1 \\
Leishmaniosis test & 2 & 0 & 0 & 6 & 0 \\
Contamination by & 0 & 0 & 0 & 2 & 0 \\
proteinaceous material & 0 & 0 & 0 & 0 & 1 \\
Contamination by fat material & 0 & 0 & 0 & 2 & 0 \\
Animal in heat & $\mathbf{1 7 1}$ & $\mathbf{8 7}$ & $\mathbf{2 5}$ & $\mathbf{6 3}$ & $\mathbf{4 6}$ \\
Total & & &
\end{tabular}

Table 3. Factors leading to the cancellation of canine cytopathological exams at the HV-UNESP-Botucatu from 2012 to 2016.

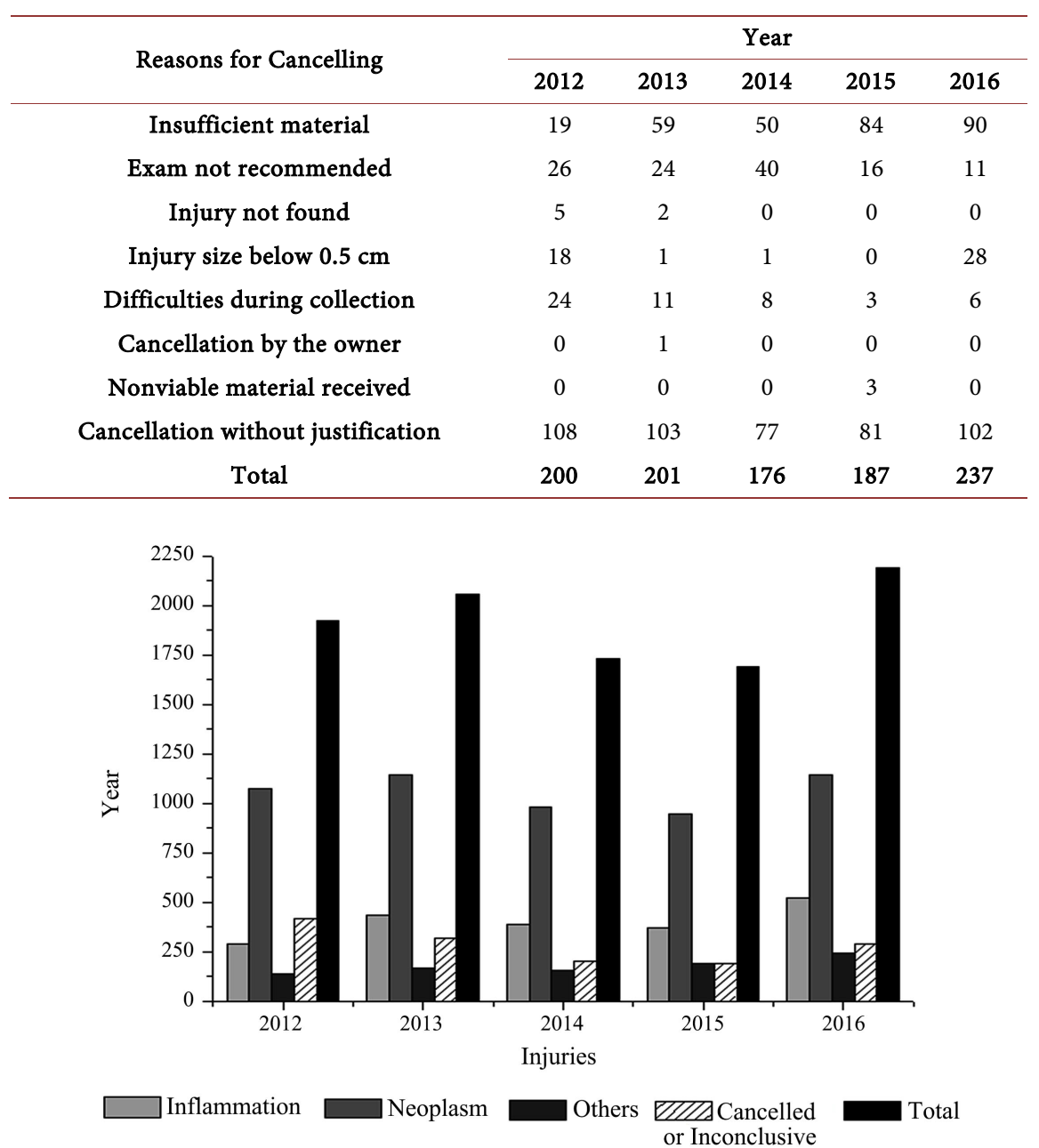

Figure 1. Classification of the injuries diagnosed in dogs through FNA in inflammation, neoplasm or others, as well as the number of cancelled or inconclusive exams at the HV-UNESP-Botucatu, from 2012 to 2016. 
of necrosis" and "negative for lymphoid tissue". Necrotic tissue may both be one of the factors reducing the accuracy of the FNA, as not enough viable material was exposed, and one of the malignancy criteria in neoplastic processes, which means that the disorganized growth, infiltrative character and high index of cellular multiplication may lead to an exaggerated disproportion between the tumoral parenchyma and the vascular stroma, resulting in extensive necrotic areas. In cases with lingering doubts regarding the classification of the process, complementary examinations should be requested [16] [32] [36] [37] [38].

Likewise, inflammatory processes may both reduce the tissue sample and be one of the malignancy criteria, since the inflammatory cells release potent soluble mediators that regulate cell survival and proliferation, angiogenesis, tissue remodeling, metabolism and genomic integrity.

The cytopathologist should classify the process as "acute/chronic inflammatory process" and, if there are suspicions that the inflammation could be masking a more severe profile, the ideal course of action creating strategies to improve the clinical profile and repeating the puncture after the treatment runs its course and the inflammatory process regresses.

Cases of "negative for neoplasm" presented rates of $39.18 \%$ and $44.83 \%$ respectively in 2012 and 2013, with a marked reduction in the following years (12\%, 3.17\% and $2.17 \%)$. This highlights the importance of FNA to identify neoplasms or discard that diagnosis, although it is important for the cytopathologist to classify the injury even when neoplastic processes are discarded. Many obstacles can be easily overcome if the characteristics of the injury are recorded properly, including the macroscopic characteristics, location, size, consistence and appearance, so that they can be compared with the microscopic findings and clinical suspicions [3] [17] [25] [37].

Cases recorded as "undetermined diagnosis" and "negative for neoplasm" have wrong classifications since all exams conducted properly should present a record of the cellular characteristics and their conformation, as well as assess the microenvironment, describing and classifying the findings according to the pathological nomenclature.

Therefore, the final cytopathological classification should be clear even if it is not definitive in order to point towards a suspicion of malignancy or other pathological processes. Definitive diagnoses should be made with the approval of a supervisor who has a mastery of the technique or through complementary examinations, such as molecular biology, cytological and histochemical markers and histopathological assays, since mistakes in this scenario could lead to False Positive or False Negative results, leading to wrong treatments, additional costs, overload of the services involved, frustration of the professionals involved and, more importantly, discomfort to the patient, as well as often causing the animal to be euthanized [39] [40] [41].

Cases recorded as "difficulties during collection" presented rates of $12 \%$, $5.47 \%, 4.55 \%, 1.6 \%$ and $2.53 \%$ from 2012 to 2016 , which may be related both 
with difficulties when restraining the animal and with the macroscopic characteristics of the injury. Likewise, in cases of "injury size below $0.5 \mathrm{~cm}$ " $(9 \%, 0.5 \%$, $0.57 \%, 0 \%$ and $11.81 \%$ respectively from 2012 to 2016 ) and "injury not found" (2.5\% in 2012 and $1 \%$ in 2013), it is difficult conduct the puncture in samples smaller than $0.5 \mathrm{~cm}$ or when the procedure is not indicated for the injury in question.

Regarding restraining the animals, there may be some difficulties with uncooperative owners after explaining the purpose of the procedure and how it works, or due to inexperienced veterinarians having difficulties when aspirating the material. As an alternative, the animal may be lightly sedated. Regarding the injury, it may present low exfoliation potential due to the histopathological characteristics of the tissue or the difficulties may be caused by its anatomical location. For the latter, the suggested procedure is a combination of FNA with imaging techniques in order to ensure correct positioning and viable samples. At any rate, micro and small injuries should be duly mapped and diagnosed so that they can be correctly staged and the prognosis can be established [39] [40].

Cases recorded as "exam not recommended" oscillated during the years, presenting rates of $13 \%, 11.94 \%, 22.73 \%, 8.56 \%$ and $4.64 \%$ from 2012 to 2016 . The classification encompasses two situations: when the cytopathologist employs a method that is not adequate for the macroscopic characteristics of the injury and for the condition of the animal; or when the cytopathologist considers of utmost importance that complementary exams be conducted, including biopsy or surgical removal for histopathological assay. Therefore, it is important that not only cytopathologists, but all veterinarians, know how to properly request exams, as described in several studies [7] [25] [35] [37].

Cases recorded as "contamination by blood" presented rates of $0.58 \%, 1.15 \%$, $36 \%, 57.14 \%$ and $73.91 \%$ from 2012 to 2016 . As is the case with necrosis and inflammation, the presence of erythrocytes may be considered a contaminant, in which case the sample should be discarded, or a criterium to differentiate benignant and malignant processes. However, in most cases, the presence of erythrocytes is caused by inadequate collection [3] [4] [38].

Cases recorded as "contamination by proteinaceous material" presented a rate of $3.17 \%$ in 2015 . It is an important particularity of the mammary gland that hinders the efficacy of the diagnosis. In addition, the presence of milk is not uncommon considering that these are important predisposition markers, together with pseudocyesis and hormonal influence. During the same year, there were two cases classified as "animal in heat", which means that erythrocytes and desquamation cells were detected, which together with the action of hormones hinders the diagnosis, which should be repeated after the estrous cycle.

One sample presented "contamination by fat material". The presence of adipose tissue also hinders diagnostic conclusion and is considerably worse when the contamination is unknown or unintended, such as the aspiration of fat or skeletal muscle adjacent to a subcutaneous mass [41]. 
In $2015,1.6 \%$ of the cases were classified as "nonviable material received". This is of utmost importance as it involves stages that are key for sample quality, such as fixation and transportation of the sample. Therefore, the wrong choice of fixing agent or other fixing methods will not allow adequate staining, or, even when staining is indeed possible, the material has suffered environmental actions that may misrepresent the patient's profile [42].

Regarding lymphoid tissue, caution should be exercised during the evaluation and collection since some neoplasms present high risk of metastasis. Therefore, the description of the injuries should be clear and the classification should be done correctly, for instance: "acute/chronic inflammatory process", "benignant/malignant neoplastic process" and "lack of alterations". It is important to highlight the relevance of FNA combined with imaging techniques for the diagnosis, staging and handling of pulmonary diseases and deep lymph nodes. As such, the ROSE protocol ensures sample adequation, reduces the number of aspirations and the stress levels of the patient, potentializing the work of the cytopathologist and generating financial savings [31] [43] [44].

In 2012, there was one case of "cancellation by the owner", which is yet to be described in the literature. The situation refers to personal problems of the owner, such as lack of time, financial restrictions, concerns regarding the procedure or lack of confidence in the professional. Therefore, it is important that the entire procedure is conducted in a standardized way by all operators [10].

At the HV-UNESP-Botucatu, there are internal protocols in place in which the viability of the material is immediately assessed by the cytopathologist, which reduces mistakes when processing and analyzing the sample. The provisory diagnosis should be registered in the protocol and in case of divergences with the final results, the doctor or surgeon would be notified. Therefore, we believe that the inconclusive results observed are directly related with the relative inexperience of the residents and the need of the professional in charge to aid in the diagnosis. This is common when the cytopathology analysis is outsourced, since the cytopathologist is not the one collecting the material and it is not uncommon for the operator to be unaware of the adequate technique for each situation [1] [17] [19].

In addition, for each cytology requisition, multiple collections should be made according to the size of the injury and in cases of multiple nodules, when there is the possibility of more than one diagnosis. Therefore, supposing that each requisition corresponds to one animal and that this animal may present more than one injury, multiple collections should be made to account for the possibility of multiple diagnoses. This type of situation is resolved by training the professionals and developing a standard for all stages of the cytopathological assay.

The Cytopathology Service at the HV-UNESP-Botucatu has adequate infrastructure to apply the ROSE protocol, including a complete Pathology Outpatient Clinic, experienced professionals able to train residents and interns, availability of materials for the study (literature, microscopes and prepared slides), and 
a high volume of cytopathology assays. As such, a standard operational procedure should be applied in order to reach excellence in our cytopathological diagnosis.

It would be ideal that residents in other services and departments of the Veterinary Hospital also be trained regarding these indications, requirements and diagnostic associations, as well as advantages and disadvantages. In addition, when analyzing the date, we strived to understand what factors prevented a successful diagnosis and possible way to correct them.

Finally, we believe that the implementation of a ROSE protocol based on the information and processes shown in this paper would provide alternatives for a standardized technique and, therefore, more accurate results and more gains to both the professionals and the patients. As shown in Table 4, the protocol provides plentiful financial benefits and improves the quality of the exam.

Therefore, a ROSE Protocol for Veterinary Medicine was outlined (Annex 1, ROSE Model adapted for Mammary Tumors in Dogs) containing the identification of the tumor and of the patient, anamnesis, clinical examination, macroscopic and microscopic characterization, diagnosis and treatment.

\section{Conclusions}

The FMVZ-UNESP Veterinary Hospital at Botucatu, Brazil, has a quality protocol, but the considerable number of cancelled and inconclusive exams means that the ROSE protocol could be implemented, as it has proven to be a viable alternative for cytopathological diagnosis even in university hospitals where the qualification of the professionals is an on-going process.

The expected gains with the exploration of the methodology and standardization of the ROSE protocol for Veterinary Medicine include the on-site evaluation of samples with speed, precision and quality, making the diagnosis quicker

Table 4. Differences with and without the ROSE protocol in services conducting FNA.

\begin{tabular}{ll}
\hline With ROSE & Without ROSE \\
\hline Protocol (guideline) & $\begin{array}{l}\text { Individual procedures according to the } \\
\text { operator }\end{array}$ \\
Defined quality control & $\begin{array}{l}\text { No quality control or individual quality } \\
\text { control }\end{array}$ \\
Guarantee of adequate material & $\begin{array}{l}\text { Indefinite adequacy } \\
\text { No repeated collections }\end{array}$ \\
Higher cellular detail & $\begin{array}{l}\text { Possible repeated collections and in conclusion } \\
\text { Lower cellular detail }\end{array}$ \\
Reliable cytopathological classification & Low reliability in cytopathological classification \\
Initial evaluation & No initial evaluation \\
Allows interprofessional interaction & No interprofessional interaction \\
Saves resources and time & Higher costs due to repeated procedures and in \\
\end{tabular}


and establishing a therapeutic protocol that is repeatable and understandable. That is, it establishes a quality standard in the three phases of clinical examinations (pre-analytical, analytical and post-analytical). In the pre-analytical phase, the ROSE protocol enables cost reduction and repeat sampling. In the Analytical, it reduces exams and in the post-analytical, wrong treatment is reduced, which ensures the patient's well-being and better prognosis.

This, in turn, would reduce costs and the number of inadequate samples, repetitions and wrong treatments, protecting the well-being and the prognosis of the patient. In addition, it would lead to higher specificity in the training of the professionals involved through the oversight of qualified professionals. Therefore, with the ROSE Protocol outlined for Veterinary Medicine, the adverse situations are limited and mistakes are reduced. In addition, the establishment of a standardized way to collect the samples aids in the interpretation and classification of the injury, assuring that the collected material is adequate not only for triage, but for definitive diagnoses, complementary examinations and prognostic examinations. This increases the maximum potential for FNAP in the field of Veterinary Medicine through the establishment of a quality control protocol.

\section{Funding Information}

Investigative and Comparative Pathology Laboratory.

\section{Conflicts of Interest}

The authors declare no conflicts of interest.

\section{References}

[1] Molinaro, E.M., Caputo, L.F.G. and Amendoeira, M.R.R. (2009) Concepts and Methods for the Training of Professionals in Health Laboratories. Fiocruz, EPSJV, IOC, Rio de Janeiro.

[2] Berlitz, F.A. (2010) Quality Control in the Clinical Laboratory: Aligning Process Improvement, Patient Reliability and Safety. Jornal Brasileiro de Patologia e Medicina Laboratorial, 46, 353-363. https://doi.org/10.1590/S1676-24442010000500003

[3] Pierini, A., Millanta, F., Zanforlin, R., Vannozzi, I. and Marchetti, V. (2017) Usefulness of Cytologic Criteria in Ultrasound-Guided Fine-Needle Aspirates from Subcentimeter Canine Mammary Tumors. Journal of Veterinary Diagnostic Investigation, 29, 869-873. https://doi.org/10.1177/1040638717718886

[4] Dolka, I., et al. (2018) Diagnostic Efficacy of Smear Cytology and Robinson's Cytological Grading of Canine Mammary Tumors with Respect to Histopathology, Cytomorphometry, Metastases and Overall Survival. PLoS ONE, 13, e0191595. https://doi.org/10.1371/journal.pone.0191595

[5] Kamiguchi, I.E., et al. (2016) Mammary Neoplasms in Female Dogs: Identification of Cytopathological Criteria for Malignancy. Journal of Cytology and Histology, 7, 392.

[6] Mazari, B., Qoku, A. and Dhaskali, L. (2018) Study of Mammary Carcinoma in a Dog and Its Relationship with Cutaneus Metastasis. ANGLISTICUM. Journal of the Association for Anglo-American Studies, 7, 46-52.

https://doi.org/10.5281/zenodo.1186434 
[7] Audrey, B., Alexis, D., Andrea, V., Julio, O. and Freddy, P.-P. (2017) Processing and Characterization of Canine Mixed Mammary Tumor Using Transmission Electron Microscopy. Microscopy Research and Technique, 80, 1229-1233. https://doi.org/10.1002/jemt.22921

[8] Cascone, A.M., et al. (2017) Fine-needle Cytology in the Follow-up of Breast Carcinoma. Aging Clinical and Experimental Research, 29, 167-171.

https://doi.org/10.1007/s40520-016-0645-Z

[9] Murussi, M., Pereira, C.E.F.N., Assis Brasil, B.M.A. and Tschiedel, B. (2001) Punção Aspirativa de Tireóide com Agulha Fina em Um Hospital Geral: Estudo de 754 Punções. Arquivos Brasileiros de Endocrinologia \& Metabologia, 45, 6. https://doi.org/10.1590/S0004-27302001000600012

[10] Eburnio, E., Correia Neiva, F.A., de Sanctis, P., Gil Mazzante, N.M. and Sousa Rocha, N. (2018) Sensitivity of the ROSE Protocol in the Evaluation of Canine Breast Cancer-A Literature Review. Advances in Biomedical Sciences, 3, 55-63.

http://www.openscienceonline.com/author/download?paperId $=4678 \&$ stateId $=8000$ \&fileType $=3$

[11] Li, C.L., Sun, W.W., Feng, J., Cao, J. and Zhang, P. (2016) Utilization of Rapid on-Site Evaluation (ROSE) in Transbronchial Needle Aspiration (TBNA), It is Not a Simple Statistical Issue. International Journal of Clinical and Experimental Medicine, 9, 13298-13308.

[12] Field, A.S. and Geddie, W.R. (2016) Role of Fine Needle Aspiration Biopsy Cytology in the Diagnosis of Infections. Diagnostic Cytopathology, 44, 1024-1038. https://doi.org/10.1002/dc.23568

[13] Collins, B.T. (2013) Telepathology in Cytopathology: Challenges and Opportunities. Acta Cytologica, 57, 221-232. https://doi.org/10.1159/000350718

[14] Santos, R.S., et al. (2018) Punção aspirativa por agulha guiada por ultrassom endobrônquico (EBUS-TBNA) no diagnóstico de lesões do mediastino. Einstein, São Paulo.

[15] Cassali, G.D., et al. (2017) Consensus for the Diagnosis, Prognosis and Treatment of Canine Mammary Tumors. Brazilian Journal of Veterinary Pathology, 4, 153-180.

[16] Santos, N., Veiga, P. and Andrade, R. (2011) Importance of History and Physical Examination for the Care of Nurses. Revista Brasileira de Enfermagem, 64, 355-358. https://doi.org/10.1590/S0034-71672011000200021

[17] Zucarri, D.A.P.C., Santana, A.E. and Rocha, N.S. (2001) Correlação entre a citologia aspirativa por agulha fina e a histologia no diagnóstico de tumores mamários de cadelas. Brazilian Journal of Veterinary Research and Animal Science, 38, 38-41. https://doi.org/10.1590/S1413-95962001000100007

[18] Cassali, G.D., et al. (2014) Consensus for the Diagnosis, Prognosis and Treatment of Canine Mammary Tumors-2013. Brazilian Journal of Veterinary Pathology, 7, 38-69. http://bjvp.org.br/wp-content/uploads/2015/07/DOWLOAD-FULL-ARTICLE-1-20 881_2014_7_31_6_23.pdf

[19] Cabral, L.A. (2017) Terapêutica clínico-cirúrgica de neoplasias mamárias em cadelas. Revista Científica do curso de Medicina Veterinária-FACIPLAC, 4, 16-30. http://revista.faciplac.edu.br/index.php/Revet/article/view/317

[20] Magalhães, A.M., Ramadinha, R.R., Barros, C.S.L. and Peixoto, P.V. (2014) Comparative Study between Cytopathology and Histopathology in the Diagnosis of Canine Neoplasias. Pesquisa Veterinária Brasileira, 21, 23-32. https://doi.org/10.1590/S0100-736X2001000100006

[21] Wilmsen, M., Tremori, T.M. and Rocha, N.S. (2016) Retrospective Study of 33 Dogs 
Diagnosed with Osteosarcoma (OSA) by Fine Needle Aspiration Cytology (FNAC) at FMVZ Vet Hospital, UNESP-Botucatu, SP. Annual Research \& Review in Biology, 9, 1-8. https://doi.org/10.9734/ARRB/2016/22707

[22] Ferreira, J.S. (2015) Cytology Application in the Diagnosis of Infectious Diseases in Domestic Animals: Review. Ciência Animal, 25, 18-24. http://www.uece.br/cienciaanimal/dmdocuments/palestra02_p18_24.pdf

[23] Ventura, R.F.A., Colodel, M.M. and Rocha, N.S. (2012) Cytological Examination in Veterinary Medicine: Retrospective Study of 11,468 Cases (1994-2008). Pesquisa Veterinária Brasileira, 32, 1169-1173. https://doi.org/10.1590/S0100-736X2012001100016

[24] Collins, J.A., Novak, A., Ali, S.Z. and Olson, M.T. (2013) Cytotechnologists and on-Site Evaluation of Adequacy. Korean Journal of Pathology, 47, 405-410.

[25] Feliciano, M.A.R., et al. (2012) Estudo Clínico, Histopatológico e Imunoistoquímico de Neoplasias Mamárias em Cadelas. Arquivo Brasileiro de Medicina Veterinária e Zootecnia, 64, 1094-1100. https://doi.org/10.1590/S0102-09352012000500002 http://www.scielo.br/pdf/abmvz/v64n5/v64n5a02.pdf

[26] Santos, G.C., Ko, H.M., Saieg, M.A. and Geddie, W.R. (2013) "The Petals and Thorns" of ROSE (Rapid on-Site Evaluation). Cancer Cytopathology, 121, 4-8. https://doi.org/10.1002/cncy.21215

[27] Collins, B.T., Garcia, T.C. and Hudson, J.B. (2016) Rapid on-Site Evaluation Improves Fine-Needle Aspiration Biopsy Cell Block Quality. Journal of the American Society of Cytopathology, 5, 37-42. https://doi.org/10.1016/j.jasc.2015.06.005

[28] Iglesias-Garcia, J., et al. (2011) Influence of On-Site Cytopathology Evaluation on the Diagnostic Accuracy of Endoscopic Ultrasound-Guided Fine Needle Aspiration (EUS-FNA) of Solid Pancreatic Masses. American Journal of Gastroenterology, 106, 1705-1710. https://doi.org/10.1038/ajg.2011.119

[29] Farrell, J.M., et al. (2018) Efficacy of Telecytopathology for Preliminary Assessment of Fine-needle Aspirations Performed at a Remote Facility. Journal of the American Society of Cytopathology, 7, 22-30. https://doi.org/10.1016/j.jasc.2017.10.001

[30] Lin, O. (2018) Telecytology for Rapid on-Site Evaluation: Current Status. Journal of the American Society of Cytopathology, 7, 1-6.

https://doi.org/10.1016/j.jasc.2017.10.002

[31] Sung, S., et al. (2018) Molecular Testing on Endobronchial Ultrasound (EBUS) Fine Needle Aspirates (FNA): Impact of Triage. Diagnostic Cytopathology, 46, 122-130. https://doi.org/10.1002/dc.23861

[32] Da Silva, M.C., et al. (2016) Conhecimento de tutores de cães sobre tumor de mama em cadelas. Acta Veterinária Brasileira, 10, 186-189.

[33] INCA-Instituto Nacional do Câncer (2016) Estimativa Incidência de Câncer no Brasil, 2016.

https://www.passeidireto.com/arquivo/35932196/estimativa-2016-v11-pdf

[34] INCA-Instituto Nacional do Câncer (2018) Estimativa Incidência de Câncer no Brasil, 2018. http://www1.inca.gov.br/estimativa/2018/estimativa-2018.pdf

[35] Tani, E., Fuentes-Martinez, N. and Skoog, L. (2017) A Review of the Use of Fine-Needle Aspiration Biopsy of Mammary Tumors for Diagnosis and Research. Acta Cytologica, 61, 305-315. https://doi.org/10.1159/000477373

[36] Ravaioli, S., et al. (2017) Easily Detectable Cytomorphological Features to Evaluate during ROSE for Rapid Lung Cancer Diagnosis: From Cytology to Histology. Oncotarget, 8, 11199-11205. https://doi.org/10.18632/oncotarget.13204 
[37] Tambouret, R.H., et al. (2014) Cytopathology and More. FNA Cytology Rapid On-Site Evaluation-How Practice Varies. Cap Today, 1-5.

https://www.captodayonline.com/fna-cytology-rapid-on-site-evaluation-how-practi ce-varies/

[38] Wong, R.W.M., et al. (2014) The Utility of Rapid On-Site Evaluation on Endobronchial Ultrasound Guided Transbronchial Needle Aspiration: Does It Make a Difference? Journal of Respiratory Medicine, 2014, Article ID: 245974. https://doi.org/10.1155/2014/245974

[39] Rossi, E.D., Wong, L.Q., Bizzarro, T., Petrone, G., Mule, A., Fadda, G., et al. (2016) The Impact of FNAC in the Management of Salivary Gland Lesions: Institutional Experiences Leading to a Risk-Based Classification Scheme. Cancer Cytopathology, 124, 388-396. https://doi.org/10.1002/cncy.21710

[40] Masood, S., Vass, L., Ibarra, J.A., Ljung, B.-M., Stalsberg, H., Eniu, A., et al. (2008) Breast Pathology Guideline Implementation in Low- and Middle-Income Countries. Cancer, 113, 2297-304. https://doi.org/10.1002/cncr.23833

[41] Kocjan, G., Chandra, A., Cross, P., Denton, K., Giles, T., Herbert, A., et al. (2009) BSCC Code of Practice-Fine Needle Aspiration Cytology. Cytopathology, 20, 283-296. https://doi.org/10.1111/j.1365-2303.2009.00709.x

[42] Araújo, M.R., Campos, L.C., Ferreira, E. and Cassali, G.D. (2015) Quantitation of the Regional Lymph Node Metastatic Burden and Prognosis in Malignant Mammary Tumors of Dogs. Journal of Veterinary Internal Medicine, 29, 1360-1367. https://doi.org/10.1111/jvim.13576

[43] Hughers, J.R., Szladovits, B. and Drees, R. (2019) Abdominal CT Evaluation of the Liver and Spleen for Staging Mast Cell Tumors in Dogs Yields Nonspecific Results. Veterinary Radiology \& Ultrasound, 60, 306-315. https://doi.org/10.1111/vru.12717

[44] Powell, R. (2018) Obtaining Good Quality Samples for Cytology. Practice, 40, 141-146. https://doi.org/10.1136/inp.k1815 


\section{Annex 1: ROSE Model Adapted for Mammary Tumors in Dogs}

Profile of Mammary Tumors in Dogs and Cost-Benefit Ratio of ROSE Before Puncture With and Without Aspiration Veterinary Cytology Outpatient Clinic-FMVZ-Botucatu, Brazil

Animal:

General Condition: ( ) Good ( ) Regular ( ) Weak

Estrus Cycle: ( ) Regular ( ) Irregular Tipe:

Number of Births: ( ) None ( ) One ( ) Two ( ) >3

Pseudopregnancy: ( ) Yes ( ) No

Abortion: ( ) Yes ( ) No

Uterine Alterations (Secretions) ( ) Yes

Hormones: ( ) Contraceptive ( ) Abortive ( ) None ( ) Does not know

Type: Date: ( ) Fast ( ) Slow

Evolution of Injuries:

Action: ( ) Surgery ( ) Other

Mammary Glands:

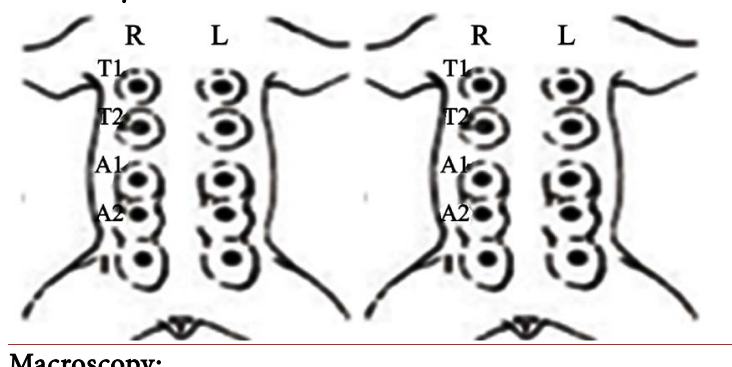

\section{Macroscopy:}

Size and Location of Injuries: ( )

( )__ $\times \ldots$

Consistence: ( ) Firm ( ) Soft ( ) Mixed ( ) Cystic

Tumor: ( ) Mobile ( ) Immobile

Mammary Glands (Which ones?

( ) No alteration ( ) Ulcers ( ) Edema ( )

Reddish Aspect ( ) Absent ( ) Others

Secretions: ( ) Yes ( ) No

Involved Lymph Nodes/Which ones: ( ) Yes ( ) No

Cytopathological:

Clinical TNM:

Imaging Diagnosis:

Number of Sample Collections/Identification:

Viability: ( ) Adequate ( ) Inadequate

Directions to the Doctor: ( ) Inflammation ( ) Benignant Neoplasm ( ) Malignant Neoplasm ( ) Others

Preliminary Result: C1-C5:

(C1, inadequate sample; $\mathrm{C} 2$, benignant cytology; $\mathrm{C} 3$, atypical cytology, probably benignant; $\mathrm{C} 4$, suspect, but not of malignancy; or C5, malignant)

Surgical Indication: ( ) Yes ( ) No Date of Surgery: ___ ___ _

Histopathological ( ) Yes ( ) No ________ Complementary Examination:

Pathological TNM ( ) Yes ( ) No

Notes

Final Diagnosis B1-B5:

B1, normal tissue; B2, benignant; B3, atypical; B4, suspect, but not of malignancy; or B5, Malignant

Pathological Classification:

Person in Charge:

Date 